\title{
An ASKAP Search for a Radio Counterpart to the First High-significance Neutron Star- Black Hole Merger LIGO/Virgo S190814bv
}

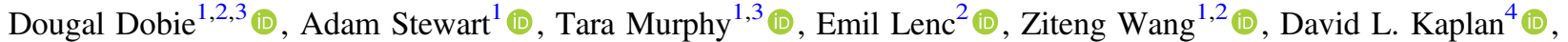

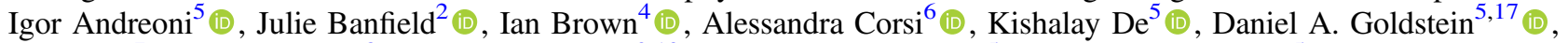 \\ Gregg Hallinan ${ }^{7}$ (D), Aidan Hotan $^{8}$, Kenta Hotokezaka ${ }^{9,10}$ (D), Amruta D. Jaodand ${ }^{5}$ (D), Viraj Karambelkar ${ }^{5}$, Mansi M. Kasliwal ${ }^{5}$ (D), \\ David McConnell ${ }^{2}$, Kunal Mooley ${ }^{7}$ (1), Vanessa A. Moss ${ }^{1,2}$ (i), Jeffrey A. Newman ${ }^{11,12}$ (1), Daniel A. Perley ${ }^{13}$ (10, \\ Abhishek Prakash $^{14}$ (10), Joshua Pritchard ${ }^{1}$ (D), Elaine M. Sadler ${ }^{1,2}$ (1), Yashvi Sharma ${ }^{5}$, Charlotte Ward ${ }^{15}$, Matthew Whiting ${ }^{2}$ (D), and \\ Rongpu Zhou ${ }^{16}$ (1) \\ ${ }^{1}$ Sydney Institute for Astronomy, School of Physics, University of Sydney, Sydney, New South Wales 2006, Australia; ddob1600@uni.sydney.edu.au, \\ tara.murphy@sydney.edu.au \\ 2 ATNF, CSIRO Astronomy and Space Science, P.O. Box 76, Epping, New South Wales 1710, Australia; \\ ${ }^{3}$ ARC Centre of Excellence for Gravitational Wave Discovery (OzGrav), Hawthorn, Victoria, Australia \\ ${ }^{4}$ Center for Gravitation, Cosmology, and Astrophysics, Department of Physics, University of Wisconsin-Milwaukee, P.O. Box 413, Milwaukee, WI 53201, USA \\ ${ }^{5}$ Division of Physics, Mathematics, and Astronomy, California Institute of Technology, Pasadena, CA 91125, USA \\ ${ }^{6}$ Department of Physics and Astronomy, Texas Tech University, Box 1051, Lubbock, TX 79409, USA \\ ${ }^{7}$ Cahill Center for Astronomy \& Astrophysics, Caltech, Pasadena CA, USA \\ ${ }^{8}$ ATNF, CSIRO Astronomy and Space Science, P.O. Box 1130, Bentley, WA 6102, Australia \\ ${ }^{9}$ Department of Astrophysical Sciences, Princeton University, 4 Ivy Lane, Princeton, NJ 08544, USA \\ ${ }^{10}$ Research Center for the Early Universe, Graduate School of Science, University of Tokyo, Bunkyo-ku, Tokyo 113-0033, Japan \\ ${ }^{11}$ Department of Physics and Astronomy, University of Pittsburgh, 3941 O'Hara Street, Pittsburgh, PA 15260, USA \\ ${ }^{12}$ Pittsburgh Particle Physics, Astrophysics, and Cosmology Center (PITT PACC), Pittsburgh, PA 15260, USA \\ ${ }^{13}$ Astrophysics Research Institute, Liverpool John Moores University, IC2, Liverpool Science Park, 146 Brownlow Hill, Liverpool L3 5RF, UK \\ ${ }^{14}$ IPAC, California Institute of Technology 1200 E California Boulevard, Pasadena, CA 91125, USA \\ ${ }_{15}$ Department of Astronomy, University of Maryland, College Park, MD 20742, USA \\ ${ }^{16}$ Lawrence Berkeley National Laboratory, 1 Cyclotron Road, Berkeley, CA 94720, USA \\ Received 2019 October 29; revised 2019 November 15; accepted 2019 November 20; published 2019 December 6
}

\begin{abstract}
We present results from a search for a radio transient associated with the LIGO/Virgo source S190814bv, a likely neutron star-black hole (NSBH) merger, with the Australian Square Kilometre Array Pathfinder. We imaged a $30 \mathrm{deg}^{2}$ field at $\Delta T=2,9$, and 33 days post-merger at a frequency of $944 \mathrm{MHz}$, comparing them to reference images from the Rapid ASKAP Continuum Survey observed 110 days prior to the event. Each epoch of our observations covers $89 \%$ of the LIGO/Virgo localization region. We conducted an untargeted search for radio transients in this field, resulting in 21 candidates. For one of these, AT2019osy, we performed multiwavelength follow-up and ultimately ruled out the association with S190814bv. All other candidates are likely unrelated variables, but we cannot conclusively rule them out. We discuss our results in the context of model predictions for radio emission from NSBH mergers and place constrains on the circum-merger density and inclination angle of the merger. This survey is simultaneously the first large-scale radio follow-up of an NSBH merger, and the most sensitive widefield radio transients search to-date.
\end{abstract}

Unified Astronomy Thesaurus concepts: Gravitational waves (678); Radio astronomy (1338); Transient sources (1851); Radio transient sources (2008)

\section{Introduction}

On 2019 August 14 the LIGO and Virgo collaborations detected the compact binary merger S190814bv ${ }^{18}$ with the LIGO Livingston (L1), LIGO Hanford (H1), and Virgo (V1) gravitational wave detectors (LIGO Scientific Collaboration \& Virgo Collaboration et al. 2019a). The event was classified as a neutron star-black hole (NSBH) merger, where the lighter component has a mass $<3 M_{\odot}$, and the heavier component has a mass $>5 M_{\odot}$ (LIGO Scientific Collaboration \& Virgo Collaboration et al. 2019b). The accuracy of this classification is dependent on the physical upper limit for neutron star mass, which is not well constrained, but may be less than the above definition (Cromartie et al. 2019; Zhang et al. 2019). The probability of there being matter outside the remnant object is

\footnotetext{
${ }^{17}$ Hubble Fellow.

18 https://gracedb.ligo.org/superevents/S190814bv/view/
}

$<1 \%$ (LIGO Scientific Collaboration \& Virgo Collaboration et al. 2019a); therefore, the expected nature of any electromagnetic radiation from the merger (if any) is unclear.

The preferred skymap (LALInference.vl.fits.gz) has a $90 \%$ localization region of $23 \mathrm{deg}^{2}$ and a sky-averaged distance estimate of $267 \pm 52 \mathrm{Mpc}$. High-energy observations (Kocevski et al. 2019; Molkov et al. 2019; Palmer et al. 2019; Pilia et al. 2019; Sugizaki et al. 2019) find no evidence for a coincident short gamma-ray burst (GRB). Optical observations found numerous candidate counterparts that have since been ruled out with further photometric and spectroscopic observations (Andreoni et al. 2019).

While the low probability of remnant matter (LIGO Scientific Collaboration \& Virgo Collaboration et al. 2019b) may suggest that the merger produced no electromagnetic counterpart, the lack of optical counterparts may also be explained by intrinsic factors such as inclination angle, mass ratio, remnant lifetime or a lack of polar ejecta (Kasen et al. 


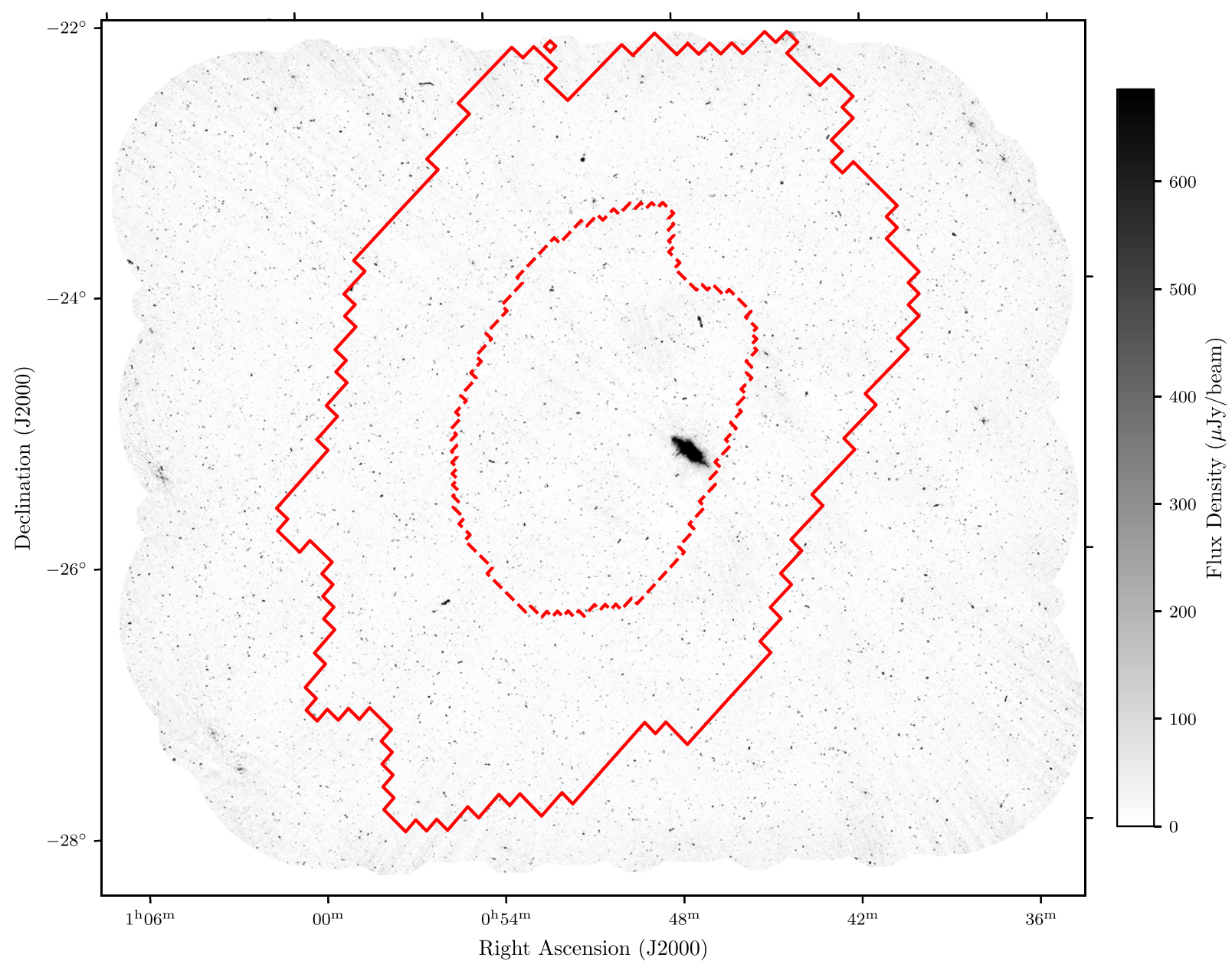

Figure 1. ASKAP image of the localization region of S190814bv centered on 00:50:37.5, -25:16:57.371 observed 2 days post-merger. The 30 deg $^{2}$ field of view covers $\sim 89 \%$ of the localization region, with $50 \%(90 \%)$ contours shown in red dashed (solid) lines. The large object near the center of the image is the radio-emitting starburst galaxy NGC 253. Note: there is a secondary lobe of the localization toward the southeast that is outside the ASKAP footprint.

Table 1

Details of our ASKAP Observations for Each Scheduling Block ID (SBID)

\begin{tabular}{|c|c|c|c|c|c|c|c|}
\hline Epoch & SBID & $\begin{array}{c}\text { Start } \\
\text { (UTC) }\end{array}$ & $\begin{array}{l}\text { Int. Time } \\
\text { (h:m:s) }\end{array}$ & $\begin{array}{c}\Delta T \\
\text { (day) }\end{array}$ & $\%$ Flagged & $\begin{array}{c}\text { Sensitivity } \\
(\mu \mathrm{Jy})\end{array}$ & Beam Size \\
\hline 0 & 8582 & 2019 Apr 27 04:59:14 & $00: 15: 00$ & -110 & 26 & 270 & $10 . .2 \times 14 ! .9$ \\
\hline 1 & 9602 & 2019 Aug 16 14:10:27 & $10: 39: 25$ & 2 & 25 & 35 & $10 " .0 \times 12$ ". 3 \\
\hline 2 & 9649 & 2019 Aug 23 13:42:59 & 10:39:01 & 9 & 26 & 39 & $11 " .8 \times 12 ! " 4$ \\
\hline 3 & 9910 & 2019 Sep 16 12:08:34 & $10: 38: 42$ & 33 & 32 & 39 & $9{ }^{\prime \prime} 8 \times 12 ! " 1$ \\
\hline
\end{tabular}

Notes. All observations were carried out with $288 \mathrm{MHz}$ of bandwidth centered on a frequency of $944 \mathrm{MHz}$ and 33 of 36 antennas. Typically $26 \%$ of the data was flagged due to RFI or correlator drop-outs. The ASKAP images from our follow-up observations are available from the CSIRO ASKAP Science Data Archive ${ }^{\mathrm{a}}$ under project code AS111.

a https://casda.csiro.au/

2017), or extrinsic factors like dust obscuration. In this case, radio emission may be the only way to localize this event.

We performed follow-up of S190814bv with the Australian Square Kilometre Array Pathfinder (ASKAP; Johnston et al. 2008). In Section 3 we discuss our untargeted radio transients search. In Section 4 we summarize multiwavelength follow-up of candidate counterpart AT2019osy that was initially detected in this search.

\section{Observations and Data Reduction}

We observed a target field centered on (J2000) coordinates $\alpha=00^{\mathrm{h}} 50^{\mathrm{m}} 37^{\mathrm{s}} .5, \quad \delta=-25^{\circ} 16^{\prime} 57^{\mathrm{s}} .37$ at $\Delta T=2, \quad 9$ and 33 days post-merger with ASKAP. This target field, shown in Figure 1 at $\Delta T=2$ days, covers $89 \%$ of the skymap probability.

Table 1 gives a summary of our ASKAP observations. Data were observed using 36 beams arranged in a closepack 36 
footprint ${ }^{19}$ with beam spacing of 0.9 . The field was tracked for a nominal time of $10.5 \mathrm{hr}$ and $288 \mathrm{MHz}$ of bandwidth was recorded with a center frequency of $944 \mathrm{MHz}$. Typical sensitivity was $\sim 39 \mu \mathrm{Jy}$ with a beam size of $\sim 12^{\prime \prime}$.

We imaged the data with the ASKAPsoft pipeline version 0.24.4 (Whiting et al. 2017), using a set of parameters optimized for deep continuum fields. Each beam was imaged independently and then combined using a linear mosaic. Multifrequency synthesis with two Taylor terms was used, along with Multi-scale CLEAN using scales up to 27 pixels in size. Visibilities were weighted using Wiener preconditioning with a robustness parameter of zero. Two major cycles of selfcalibration were used to refine the antenna gain solutions derived from observations of PKS B1934-638 in each beam (see McConnell et al. 2016, for a description of the ASKAP beamforming and calibration process). We also used prerelease data from the $888 \mathrm{MHz}$ Rapid ASKAP Continuum Survey $\left(\mathrm{RACS}^{20}\right)$ as a reference epoch.

The astrometric accuracy and flux scaling of each epoch is consistent with every other epoch. The median flux ratio of compact sources for any two of the ASKAP observations is consistent with 1 within uncertainties. The median R.A. offset is $0 . " 09-0$ ". 36 and the median decl. offset is $0 . " 02-0$ ". 2 (smaller than the pixel size) with a typical standard deviation of 0.77 and 0 ". 6 respectively.

\section{Untargeted Search for Radio Transients and Variables}

To search for a radio counterpart to S190814bv, we performed an untargeted search for transients and highly variable sources using the LOFAR Transients Pipeline (TraP; Swinbank et al. 2015). We ran TraP with source detection and analysis thresholds of $5 \sigma$ and $3 \sigma$, respectively, and used the "force beam" option to constrain the Gaussian shape fit parameters for all sources to be the same as the restoring beam.

We selected candidates by identifying sources that were significant outliers in both variability metrics calculated by TraP: $\eta$, which is the weighted reduced $\chi^{2}$, and the variability index $V$ (equivalent to the fractional variability). This was done by fitting a Gaussian function to the distributions of both metrics in logarithmic space, with $\sigma$ thresholds chosen to be $\eta>1.5 \sigma_{\eta}$ and $V>1.0 \sigma_{V}$, equating to values of $\eta>2.73$ and $V>0.18$. The thresholds were adapted from Rowlinson et al. (2019), which gives approximate recall and precision rates of $90 \%$ and $50 \%$ respectively.

This resulted in 285 transient or variable candidates, which was reduced to 89 sources after manual inspection to remove imaging artifacts and components of complex extended sources.

\subsection{Analysis of Candidates for Possible Association with S190814bv}

The 89 variable sources were filtered to remove those that were not consistent with the predicted emission of S190814bv, which should not exhibit more than a single rise and decline on these timescales (Hotokezaka et al. 2016), according to the following criteria:

\footnotetext{
${ }^{19}$ For more information on ASKAP beam-forming, see https://confluence. csiro.au/display/askapsst/.

${ }^{20}$ https://www.atnf.csiro.au/content/racs
}

1. Sources that showed a decline between epochs 1 and 2 , followed by a rise between epochs 2 and 3. 41 sources were excluded.

2. Sources detected in RACS epoch 0 where epochs 1 and 2 had lower integrated flux values than epoch 0.3 sources were excluded.

We then searched the GLADE catalog (GLADE; Dálya et al. 2018 ) for galaxies in the localization volume within $20^{\prime \prime}$ (or $\sim 20 \mathrm{kpc}$ at the estimated distance of S190814bv LIGO Scientific Collaboration \& Virgo Collaboration et al. 2019b) of a variable source. We found one candidate (ASKAP J005547.4-270433) that is near 2dFGRS TGS211Z177, a cataloged galaxy with $z=0.0738$ (Colless et al. 2001). This source was the only strong candidate after epoch 2 and prior to the acquisition of epoch 3 we performed multiwavelength follow-up which we discuss in Section 4. We excluded two candidates that matched with a GLADE galaxy $>3 \sigma$ beyond the estimated distance to S190814bv $(267 \pm 52$ Mpc LIGO Scientific Collaboration \& Virgo Collaboration et al. 2019b).

We cross-matched the 42 remaining variable candidates with the Photometric Redshifts for the Legacy Surveys (PRLS) catalog (R. Zhou et al. 2019, in preparation), which is based on Data Release 8 of DESI Legacy Imaging Surveys (Dey et al. 2019). We excluded 22 variable sources that had all optical matches at distances differing by $>3 \sigma$ from the estimated distance to S190814bv. This left seven sources with at least one cross-match within the localization volume and 13 sources with no reliable distance estimate (see Table 2).

\section{Follow-up of ASKAP J005547.4-270433}

\subsection{Radio Observations}

We carried out follow-up observations of ASKAP J005547.4-270433 (hereafter AT2019osy) with the ATCA (C3278, PI: Dobie) using two $2 \mathrm{GHz}$ bands centered on 5.5 and $9 \mathrm{GHz}$ at 14,22 , and 34 days post-merger. We reduced the data using the same method as Dobie et al. (2018) using PKS B1934-638 and B0118-272 as flux and phase calibrators respectively.

We also carried out VLA observations (VLA 18B-320, PI: Frail) on 2019 August 28 and September 9. Standard 8 bit WIDAR correlator setups were used for $L$ and $S$ bands, and 3 bit setups for $C$ and $X$ bands to obtain a contiguous frequency coverage between 1 and $12 \mathrm{GHz}$. 3C48 and J0118-2141 were used as the flux and phase calibrators respectively. The data were processed using the NRAO CASA pipeline and imaged using the clean task in CASA.

A summary of our observations is given in Table 3. We find a constant flux density offset ${ }^{21}$ of $\sim 40 \%$ between the initial ATCA and VLA observations across all epochs. We therefore find no evidence for radio variability beyond the initial rise observed with ASKAP.

\subsection{Optical Observations}

We conducted optical imaging of AT2019osy with the Dark Energy Camera (DECam, Flaugher et al. 2015) on the $4 \mathrm{~m}$

\footnotetext{
${ }^{21}$ The flux densities of nearby sources and the calibrator source J0118-2141 between the ATCA and the VLA are consistent with the flux offset of $40 \%$ seen in AT2019osy. This offset can partially be explained by resolution effects, and detailed investigation of it is ongoing.
} 
Table 2

Candidate Counterparts from an Untargeted Search of the S190814bv Localization Region

\begin{tabular}{|c|c|c|c|c|c|c|c|c|c|c|}
\hline Name & $\begin{array}{l}\text { R.A. } \\
\text { (deg) }\end{array}$ & $\begin{array}{l}\text { Decl. } \\
\text { (deg) }\end{array}$ & $\begin{array}{c}S_{0} \\
(\mathrm{mJy})\end{array}$ & $\begin{array}{c}S_{1} \\
(\mathrm{mJy})\end{array}$ & $\begin{array}{c}S_{2} \\
(\mathrm{mJy})\end{array}$ & $\begin{array}{c}S_{3} \\
(\mathrm{mJy})\end{array}$ & $V_{\text {int }}$ & $\eta_{\text {int }}$ & $\begin{array}{c}\text { Offset } \\
\left({ }^{\prime \prime}\right)\end{array}$ & $z$ \\
\hline ASKAP J004033.2-233530 & 10.13813 & -23.5917 & $4.700 \pm 0.454$ & $4.517 \pm 0.062$ & $4.732 \pm 0.069$ & $6.648 \pm 0.068$ & 0.22 & 306 & $\ldots$ & \\
\hline ASKAP J004054.8-273246 & 10.22816 & -27.5463 & $<1.1$ & $0.498 \pm 0.069$ & $0.525 \pm 0.076$ & $0.272 \pm 0.078$ & 0.32 & 3.29 & 13.4 & $0.19 \pm 0.05$ \\
\hline ASKAP J004150.3-270632 & 10.45977 & -27.1090 & $<1.0$ & $0.656 \pm 0.058$ & $0.536 \pm 0.063$ & $0.436 \pm 0.064$ & 0.20 & 3.32 & $\ldots$ & $\ldots$ \\
\hline ASKAP J004424.5-265522 & 11.10216 & -26.9230 & $<1.2$ & $0.281 \pm 0.055$ & $0.437 \pm 0.060$ & $0.475 \pm 0.060$ & 0.26 & 3.26 & $\ldots$ & $\ldots$ \\
\hline ASKAP J004825.7-264137 & 12.10704 & -26.6937 & $<0.75$ & $0.384 \pm 0.053$ & $0.615 \pm 0.057$ & $0.614 \pm 0.057$ & 0.25 & 5.94 & $\ldots$ & $\ldots$ \\
\hline ASKAP J004916.8-270745 & 12.32005 & -27.1292 & $<0.88$ & $0.586 \pm 0.049$ & $0.725 \pm 0.053$ & $0.954 \pm 0.055$ & 0.25 & 12.6 & 16.8 & $0.38 \pm 0.13^{\mathrm{a}}$ \\
\hline ASKAP J005234.9-264144 & 13.14558 & -26.6956 & $<0.73$ & $0.379 \pm 0.050$ & $0.380 \pm 0.055$ & $0.226 \pm 0.054$ & 0.27 & 2.75 & $\cdots$ & $\cdots$ \\
\hline ASKAP J005304.8-255451 & 13.27001 & -25.9144 & $<1.1$ & $0.230 \pm 0.050$ & $0.375 \pm 0.054$ & $0.214 \pm 0.053$ & 0.33 & 2.75 & $\ldots$ & $\cdots$ \\
\hline ASKAP J005426.1-253833 & 13.60866 & -25.6425 & $<0.72$ & $0.274 \pm 0.053$ & $0.487 \pm 0.059$ & $0.273 \pm 0.059$ & 0.36 & 4.51 & 17.9 & $0.33 \pm 0.11$ \\
\hline ASKAP J005434.6-280235 & 13.64412 & -28.0431 & $<0.70$ & $3.399 \pm 0.097$ & $1.337 \pm 0.103$ & $1.264 \pm 0.104$ & 0.61 & 149 & 11.5 & $0.21 \pm 0.11$ \\
\hline ASKAP J005523.7-250403 & 13.84868 & -25.0675 & $<0.86$ & $0.972 \pm 0.053$ & $0.753 \pm 0.060$ & $0.669 \pm 0.060$ & 0.20 & 7.85 & $\cdots$ & $\cdots$ \\
\hline ASKAP J005547.4-270433 & 13.94764 & -27.0759 & $<0.80$ & $0.399 \pm 0.055$ & $0.598 \pm 0.059$ & $0.557 \pm 0.059$ & 0.20 & 3.45 & 0.1 & $0.0733^{\mathrm{c}}$ \\
\hline ASKAP J005606.9-255300 & 14.02875 & -25.8835 & $<0.80$ & $0.623 \pm 0.052$ & $0.899 \pm 0.059$ & $1.011 \pm 0.059$ & 0.24 & 13.3 & 9.2 & $0.26 \pm 0.14$ \\
\hline ASKAP J005618.1-273012 & 14.07556 & -27.5035 & $2.006 \pm 0.559$ & $1.770 \pm 0.066$ & $2.613 \pm 0.070$ & $2.050 \pm 0.069$ & 0.20 & 39.4 & 11.1 & $0.18 \pm 0.09$ \\
\hline ASKAP J005709.0-243659 & 14.28753 & -24.6165 & $<0.78$ & $0.890 \pm 0.054$ & $0.611 \pm 0.060$ & $0.489 \pm 0.059$ & 0.31 & 13.5 & 14.2 & $0.22 \pm 0.10$ \\
\hline ASKAP J005709.7-250751 & 14.29030 & -25.1310 & $<0.81$ & $0.654 \pm 0.054$ & $0.814 \pm 0.062$ & $0.447 \pm 0.062$ & 0.29 & 8.85 & $\ldots$ & $\ldots$ \\
\hline ASKAP J005729.6-231608 & 14.37350 & -23.2690 & $<0.98$ & $0.620 \pm 0.060$ & $0.803 \pm 0.065$ & $0.495 \pm 0.064$ & 0.24 & 5.76 & $\cdots$ & $\cdots$ \\
\hline ASKAP J005809.0-273407 & 14.53757 & -27.5688 & $<0.79$ & $0.849 \pm 0.068$ & $0.602 \pm 0.072$ & $0.552 \pm 0.073$ & 0.24 & 5.25 & $\ldots$ & $\ldots$ \\
\hline ASKAP J010004.6-231155 & 15.01934 & -23.1988 & $<0.79$ & $1.002 \pm 0.067$ & $0.767 \pm 0.073$ & $0.642 \pm 0.070$ & 0.23 & 7.15 & $\cdots$ & $\cdots$ \\
\hline ASKAP J010258.6-265119 & 15.74436 & -26.8555 & $<0.87$ & $<0.099$ & $0.261 \pm 0.091$ & $0.232 \pm 0.098$ & 0.45 & 3.75 & $\cdots$ & $\cdots$ \\
\hline ASKAP J010534.6-231604 & 16.39415 & -23.2680 & $<0.85$ & $<0.087$ & $0.485 \pm 0.140$ & $0.718 \pm 0.146$ & 0.58 & 3.36 & $\cdots$ & $\cdots$ \\
\hline
\end{tabular}

Notes. Nondetections are denoted by $3 \sigma$ upper limits based on the local noise measured by BANE (Hancock et al. 2018). The angular separation and redshift of the corresponding optical source are shown.

${ }^{\mathrm{a}}$ There are three optical sources within $20^{\prime \prime}$ of this candidate. The two closest have a photometric redshift that is inconsistent with the distance to S190814bv.

${ }^{\mathrm{b}}$ Ruled out as a counterpart.

${ }^{\mathrm{c}}$ Spectroscopic redshift. 
Table 3

Radio Observations of AT2019osy

\begin{tabular}{lccc}
\hline \hline Telescope & $\begin{array}{c}\Delta T \\
(\text { days })\end{array}$ & $\begin{array}{c}\text { Frequency } \\
(\mathrm{GHz})\end{array}$ & $\begin{array}{c}\text { Flux Density } \\
(\mu \mathrm{Jy})\end{array}$ \\
\hline ASKAP & 2 & 0.943 & $376 \pm 33$ \\
\hline ASKAP & 9 & 0.943 & $550 \pm 34$ \\
\hline VLA & 13 & 1.5 & $409 \pm 34$ \\
& & 3.0 & $301 \pm 21$ \\
& & 6.0 & $213 \pm 11$ \\
\hline ATCA & 10.0 & $187 \pm 11$ \\
& 14 & 5.0 & $369 \pm 23$ \\
& & 6.0 & $335 \pm 19$ \\
\hline ATCA & 8.5 & $307 \pm 15$ \\
& 22 & 9.5 & $278 \pm 14$ \\
\hline VLA & & 5.0 & $380 \pm 21$ \\
& & 6.0 & $293 \pm 17$ \\
& 25 & 8.5 & $234 \pm 14$ \\
\hline ASKAP & & 9.5 & $303 \pm 48$ \\
\hline ATCA & & 1.5 & $317 \pm 21$ \\
& 33 & 3.0 & $220 \pm 10$ \\
& & 6.0 & $150 \pm 10$ \\
\hline & & 0.0 & $513 \pm 34$ \\
\hline
\end{tabular}

Note. Observations with the ATCA and VLA were carried out with maximum baselines of $6 \mathrm{~km}$ and $40 \mathrm{~km}$ respectively.

Blanco telescope under NOAO program ID 2019B-0372 (PI: Soares-Santos). Images including the location of AT2019osy were taken in $i$ and $z$ bands nightly from 2019 August 15 to 18 and on 2019 August 21 (UT) and reduced in real-time (Goldstein et al. 2019). A detailed offline analysis of the subtraction images zooming in on the location around AT2019osy, reveals no robust point source at this location to a depth of $i>21.2 \mathrm{mag}$ and $z>20.0 \mathrm{mag}$ on UT 2019 August 15 (the night of the merger) increasing linearly in limiting magnitude to $i>23.5 \mathrm{mag}$ and $z>23.5 \mathrm{mag}$ on UT 2019 August 21 (consistent with independent analysis by Herner et al. 2019). We also analyzed the DECam images using The Tractor image modeling software (Lang et al. 2016) and found that a model with an exponential galaxy profile with a point source at the galaxy nucleus is required to fit the data, both before and after S190814bv. This suggests that there is no optical transient temporally coincident with S190814bv but possibly some underlying nuclear variability.

On 2019 August 22 UT, we observed AT2019osy in the near-infrared using the Wide-field Infrared Camera (WIRC; Wilson et al. 2003) with the 200 inch Hale telescope at Palomar Observatory for a total of 10 minute exposure time (De et al. 2019b). The WIRC data were reduced and stacked using a custom pipeline (De et al. 2019a). No counterpart to AT2019osy was detected down to an AB limiting magnitude of $J>21.5(5 \sigma)$.

We also obtained a spectrum of the host galaxy of AT2019osy using the Double Beam Spectrograph (Oke \&
Gunn 1982) on the Palomar 200 inch Hale Telescope (P200), which we reduced using pyraf-dbsp (Bellm \& Sesar 2016). The spectrum is dominated by red continuum that is likely primarily associated with the host galaxy; no obvious broad features are evident. We identify several narrow emission lines $(\mathrm{H} \alpha ; \quad[\mathrm{N} \mathrm{II}] \lambda \lambda 6548,6583$, [S II] $\lambda \lambda 6716,6731$, and marginal [O II] $\lambda 3727$ ) at a common redshift of 0.0733 , consistent within $2 \sigma$ of the LVC distance constraint. $\mathrm{H} \beta$ and [O III] $\lambda 5007$ are not detected in the spectrum. We measure a flux ratio of $\log$ $[\mathrm{N}$ II $\lambda 6583 / \mathrm{H} \alpha]=0.2$, indicating at least partial contribution by an active galactic nucleus (AGN; Kauffmann et al. 2003).

\subsection{X-Ray Observations}

We observed the field of AT2019osy, starting at 2019 September 23 10:30:48 UT for 20 ks with the Chandra ACIS-S instrument (S3 chip) and very faint data mode. The data were analyzed with CIAO (v 4.11; Fruscione et al. 2006) and calibration was carried out with CALDBv4.8.4.1. We reprocessed the primary and secondary data using the repro script and created X-ray images for the $0.3-8 \mathrm{keV}$ range. No sources were visible near AT2019osy (verified with both wavdetect and celldetect), with a maximum count rate of $2.85 \times 10^{-4} \mathrm{~s}^{-1}$. Assuming a neutral hydrogen column density $N_{\mathrm{H}}=1.8 \times 10^{20} \mathrm{~cm}^{-2}$ and a power-law model with index $n=1.66$ (corresponding to the observed radio spectral index of -0.4 ), this count rate yields a $0.3-8 \mathrm{keV}$ unabsorbed flux upper limit of $3.2 \times 10^{-15} \mathrm{erg} \mathrm{cm}^{-2} \mathrm{~s}^{-1}$ (as reported in Jaodand et al. 2019) or an unabsorbed luminosity of $4.2 \times 10^{40} \mathrm{erg} \mathrm{s}^{-1}$.

\subsection{Source Classification}

AT2019osy exhibits no significant radio variability beyond the initial rise and there is no evidence for a coincident optical transient. The coincident galaxy is edge-on, likely with significant dust obscuration toward the nucleus, and therefore the optical spectrum is consistent with an AGN within a starforming galaxy. The inferred radio and X-ray luminosity of AT2019osy along with the small offset from the optical centroid of 2dFGRS TGS211Z177 suggests that the source is a variable low-luminosity AGN (Ballo et al. 2012) and unrelated to S190814bv.

\section{Discussion}

\subsection{Candidate Classification}

We find 21 candidate counterparts to S190814bv above our detection threshold of $170 \mu \mathrm{Jy}$, corresponding to $\lesssim 1 \%$ of observed sources. This is consistent with the expected rate of AGN variability from Radcliffe et al. (2019), who find $\sim 2 \%$ of $\mu \mathrm{Jy}$-level sources exhibit significant variability likely attributable to the presence of an AGN. Additionally, the expected level of compact source variability caused by refractive interstellar scintillation along this line of sight is $\sim 35 \%$ (Cordes \& Lazio 2002), comparable to $V_{\text {int }}$ for all but three sources which we discuss below.

We classify ASKAP J005434.6-280235 as a variable AGN based on follow-up observations (De et al. 2019b; Dobie et al. 2019). ASKAP J010258.6-265119 is coincident centrally between two large radio lobes and hence likely associated with core emission from a radio galaxy. ASKAP J010534.6-231604 is coincident $\left(<1^{\prime \prime}\right)$ with WISE 
J010534.64-231605.5 (Cutri et al. 2012), which is likely a variable $A G N$ at a distance of $z \sim 1$ (Glowacki et al. 2017).

While we cannot conclusively rule out the sources in Table 2 as counterparts to $S 190814 \mathrm{bv}$, they are likely AGN exhibiting a combination of intrinsic and extrinsic variability. Of course, at most one candidate can be the actual counterpart, and there is nothing yet to distinguish any of these from the others. Further observations on timescales of months to years will reveal their nature.

\subsection{Radio Transient Rates}

Our follow-up of S190814bv is the most sensitive widefield radio transients search to-date, approximately an order of magnitude more sensitive compared to previous searches with comparable areal coverage (Hobbs et al. 2016) and approximately an order of magnitude more areal coverage than previous searches at comparable sensitivities (Mooley et al. 2013).

We have found four transient candidates (i.e., sources with a prior constraining nondetection) in total; the three sources discussed in Section 5.1 and ASKAP J005104.2-230852, which was ruled out as a candidate to S190814bv based on the redshift of nearby optical sources. This source is coincident $(<$ 0".6) with WISE J005104.13-230851.8, which is likely a variable AGN.

We therefore place an upper limit on the $943 \mathrm{MHz}$ radio transients surface density of $0.05 \mathrm{deg}^{-2}$ for sources above $170 \mu \mathrm{Jy}$ at $95 \%$ confidence.

\subsection{Nondetection of a Radio Afterglow from S190814bv}

Predicted radio lightcurves from NSBH mergers span a large range of flux densities and timescales (e.g., Piran et al. 2013; Lamb \& Kobayashi 2016; Bhattacharya et al. 2019). If the radio emission is dominated by the outflowing dynamical ejecta the lightcurve will peak on timescales of years, whereas if the emission is jet-dominated the lightcurve will peak at comparably lower flux densities on timescales of days to months (Hotokezaka et al. 2016). In each of these scenarios the lightcurve is also dependent on the merger energetics, circummerger density and inclination angle, each of which can change both the peak time and flux density by an order of magnitude. The merger energetics are determined by the mass ratio, the spin of the black hole (both of which are calculable from gravitational wave strain data that is yet to be released), and the unknown neutron star equation of state (Kyutoku et al. 2011; Foucart 2012).

We place a $5 \sigma$ upper limit on the $943 \mathrm{MHz}$ radio emission from $\mathrm{S} 190814 \mathrm{bv}$ of $170 \mu \mathrm{Jy}$ at $\Delta T=2,9$, and 33 days postmerger. We compute off-axis afterglow lightcurves based on a top-hat jet model (see Hotokezaka \& Piran 2015, for details) assuming an isotropic equivalent energy $E_{\mathrm{iso}}=10^{51} \mathrm{erg}$ (typical of short GRB afterglows; Fong et al. 2015), an initial jet opening angle of $\theta_{\mathrm{j}}=10^{\circ}$ and microphysics parameters $\epsilon_{e}=0.1, \epsilon_{B}=0.01$, and $p=2.2$. By comparing these lightcurves to the observed upper limits we can constrain the merger inclination angle, $\theta_{\text {obs }}$, and circum-merger density, $n$. Figure 2 shows these constraints, assuming that the merger occurred within the $89 \%$ of the localization region covered by our observations. We can rule out the part of the parameter space typically occupied by short GRBs, assuming that their inclination angle is smaller than the opening of the angle of

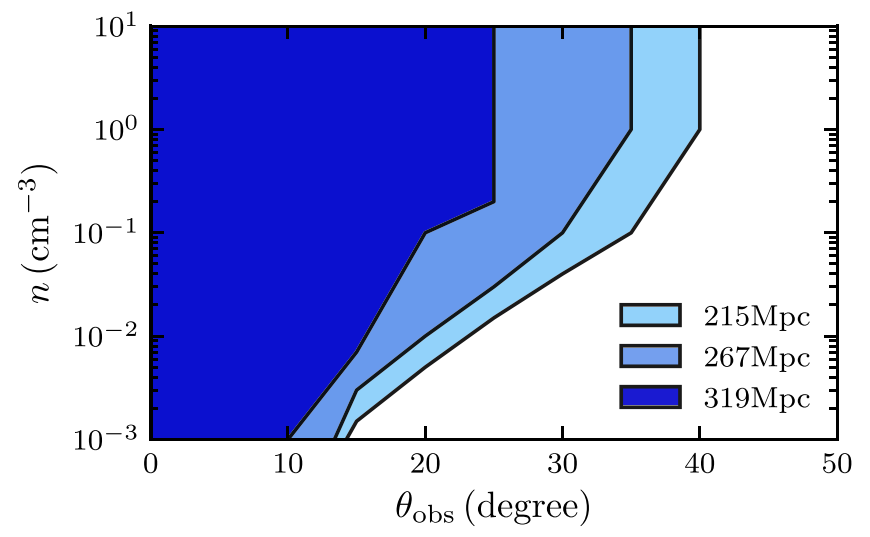

Figure 2. Radio constraints on viewing angle and circum-merger density for a merger with isotropic equivalent energy $10^{51} \mathrm{erg}$, an initial jet opening angle of $10^{\circ}$, and microphysics parameters $\epsilon_{e}=0.1, \epsilon_{B}=0.01$, and $p=2.2$, assuming that the merger occurred in the $89 \%$ of the localization region we observed. Shaded regions correspond to parts of the parameter space that are ruled out by our radio constraints for a range of distances corresponding to $1 \sigma$ either side of the median.

the jet (Fong et al. 2015). Under a more conservative assumption of the isotropic equivalent energy $\left(E_{\mathrm{iso}}=10^{50}\right.$ erg) we can only rule out a small part of the parameter space around $\theta_{\mathrm{obs}}=10^{\circ}$ and $n=1 \mathrm{~cm}^{-3}$.

In comparison, if we scale the nonthermal lightcurve of GW170817 to $943 \mathrm{MHz}$ based on a spectral index of $\alpha=-0.575$ (Mooley et al. 2018; Hajela et al. 2019) and place it at a distance comparable to $\mathrm{S} 190814 \mathrm{bv}$, we find a peak flux density of $\sim 5 \mu \mathrm{Jy}$, well below our detection threshold. We note that the nonthermal emission from GW170817 did not peak until $\sim 150$ days post-merger (Dobie et al. 2018). Further observations on timescales of months to years post-merger will enable us to place tighter constraints on the circum-merger density and inclination angle, which may be useful in improving the gravitational wave localization (Corley et al. 2019).

\section{Conclusions}

We have performed widefield radio follow-up of the NS-BH merger S190814bv with the Australian ASKAP. We cover 89\% of the sky localization with a single $30 \mathrm{deg}^{2}$ pointing centered on the localization maxima. We found 21 candidate counterparts and performed comprehensive multiwavelength follow-up of one, AT2019osy. The number of candidates is consistent with the expected rate of AGN variability. Most exhibit variability that is consistent with that expected from interstellar scintillation and are therefore unlikely to be related to S190814bv.

The nondetection of a radio counterpart allows us to place constraints on the circum-merger density, $n$, and inclination angle of the merger, $\theta_{\text {obs }}$, if it occurred within the area covered by our observations. Under the assumption of $E_{\mathrm{iso}}=10^{51} \mathrm{erg}$, $\theta_{\mathrm{j}}=10^{\circ}, \epsilon_{e}=0.1, \quad \epsilon_{B}=0.01$, and $p=2.2$, we constrain $\theta_{\mathrm{obs}}>10^{\circ}$ for all $n$ at the extreme of the probability distribution of distance to the event. We will be able to place tighter constraints on these merger parameters once inclination angle estimates from gravitational wave strain data are released publicly.

As well as probing different parameters to optical searches, radio observations of future events may detect a gravitational wave counterpart where optical follow-up is inhibited by 
observing constraints, or intrinsic properties of the merger. We have demonstrated that it is possible to perform comprehensive follow-up of gravitational wave events with ASKAP, due to its large field of view that enables a survey speed significantly faster than comparable radio facilities.

D.D. is supported by an Australian Government Research Training Program Scholarship. T.M. acknowledges the support of the Australian Research Council through grant DP190100561. D.L.K. and I.B. were supported by NSF grant AST-1816492. Parts of this research were conducted by the Australian Research Council Centre of Excellence for Gravitational Wave Discovery (OzGrav), project number CE170100004. We acknowledge support by the GROWTH (Global Relay of Observatories Watching Transients Happen) project funded by the National Science Foundation PIRE (Partnership in International Research and Education) program under Grant No. 1545949. A.C. acknowledges support from the NSF CAREER award \#1455090. D.A.G. acknowledges support from Hubble Fellowship grant HST-HF2-51408.001A. Support for Program number HST-HF2-51408.001-A is provided by NASA through a grant from the Space Telescope Science Institute, which is operated by the Association of Universities for Research in Astronomy, Incorporated, under NASA contract NAS5-26555. Development of the PRLS photometric redshift catalog used here was supported by the U.S. Department of Energy, Office of Science, Office of High Energy Physics under award number DE-SC0007914. The National Radio Astronomy Observatory is a facility of the National Science Foundation operated under cooperative agreement by Associated Universities, Inc.

The Australian SKA Pathfinder is part of the Australia Telescope National Facility which is managed by CSIRO. Operation of ASKAP is funded by the Australian Government with support from the National Collaborative Research Infrastructure Strategy. ASKAP uses the resources of the Pawsey Supercomputing Centre. Establishment of ASKAP, the Murchison Radio-astronomy Observatory and the Pawsey Supercomputing Centre are initiatives of the Australian Government, with support from the Government of Western Australia and the Science and Industry Endowment Fund. We acknowledge the Wajarri Yamatji people as the traditional owners of the Observatory site.

The Australia Telescope Compact Array is part of the Australia Telescope National Facility which is funded by the Australian Government for operation as a National Facility managed by CSIRO.

This research has made use of NASA's Astrophysics Data System Bibliographic Services. This publication makes use of data products from the Wide-field Infrared Survey Explorer, which is a joint project of the University of California, Los Angeles, and the Jet Propulsion Laboratory/California Institute of Technology, funded by the National Aeronautics and Space Administration.

Facilities: ASKAP, ATCA, VLA, Blanco, Hale, Chandra.

Software: ASKAPsoft (Whiting et al. 2017), BANE (Hancock et al. 2018), CASA (McMullin et al. 2007), pyraf-dbsp (Bellm \& Sesar 2016), The Tractor (Lang et al. 2016), TraP (Swinbank et al. 2015).

\section{ORCID iDs}

Dougal Dobie (ㄱ) https://orcid.org/0000-0003-0699-7019
Adam Stewart (1) https://orcid.org/0000-0001-8026-5903 Tara Murphy iㅣ https://orcid.org/0000-0002-2686-438X Emil Lenc (i) https://orcid.org/0000-0002-9994-1593 Ziteng Wang (i) https://orcid.org/0000-0002-2066-9823 David L. Kaplan (1) https://orcid.org/0000-0001-6295-2881 Igor Andreoni (i) https://orcid.org/0000-0002-8977-1498 Julie Banfield (1) https://orcid.org/0000-0003-4417-5374 Ian Brown 주 https://orcid.org/0000-0003-1095-8194 Alessandra Corsi (1) https://orcid.org/0000-0001-8104-3536 Kishalay De (1) https://orcid.org/0000-0002-8989-0542 Daniel A. Goldstein (1) https://orcid.org/0000-0003-3461-8661 Gregg Hallinan (1) https://orcid.org/0000-0002-7083-4049 Kenta Hotokezaka (1) https://orcid.org/0000-0002-2502-3730 Amruta D. Jaodand (1) https://orcid.org/0000-0002-3850-6651 Mansi M. Kasliwal (i) https://orcid.org/0000-0002-5619-4938 Kunal Mooley (1) https://orcid.org/0000-0002-2557-5180 Vanessa A. Moss (1) https://orcid.org/0000-0002-3005-9738 Jeffrey A. Newman (1) https://orcid.org/0000-0001-8684-2222 Daniel A. Perley (i) https://orcid.org/0000-0001-8472-1996 Abhishek Prakash (1) https://orcid.org/0000-0003-4451-4444 Joshua Pritchard (10) https://orcid.org/0000-0003-1575-5249 Elaine M. Sadler (i) https://orcid.org/0000-0002-1136-2555 Matthew Whiting (1) https://orcid.org/0000-0003-1160-2077 Rongpu Zhou (i) https://orcid.org/0000-0001-5381-4372

\section{References}

Andreoni, I., Goldstein, D. A., Kasliwal, M. M., et al. 2019, ApJ, submitted (arXiv:1910.13409)

Ballo, L., Heras, F. J. H., Barcons, X., \& Carrera, F. J. 2012, A\&A, 545, A66 Bellm, E. C., \& Sesar, B. 2016, pyraf-dbsp: Reduction pipeline for the Palomar Double Beam Spectrograph, Astrophysics Source Code Library, ascl:1602.002

Bhattacharya, M., Kumar, P., \& Smoot, G. 2019, MNRAS, 486, 5289 Colless, M., Dalton, G., Maddox, S., et al. 2001, MNRAS, 328, 1039 Cordes, J. M., \& Lazio, T. J. W. 2002, arXiv:astro-ph/0207156

Corley, K. R., Bartos, I., Singer, L. P., et al. 2019, MNRAS, 488, 4459

Cromartie, H. T., Fonseca, E., Ransom, S. M., et al. 2019, NatAs, in press (doi:10.1038/s41550-019-0880-2)

Cutri, R. M., Wright, E. L., Conrow, T., et al. 2012, yCat, 2311, 1

Dálya, G., Galgóczi, G., Dobos, L., et al. 2018, MNRAS, 479, 2374

De, K., Hankins, M. J., Kasliwal, M. M., et al. 2019a, PASP, submitted (arXiv:1910.13319)

De, K., Tinyanont, S., Nguyen, M., et al. 2019b, GCN, 25449, 1

Dey, A., Schlegel, D. J., Lang, D., et al. 2019, AJ, 157, 168

Dobie, D., Kaplan, D. L., Murphy, T., et al. 2018, ApJL, 858, L15

Dobie, D., Stewart, A. J., Wang, Z., et al. 2019, GCN, 25445, 1

Flaugher, B., Diehl, H. T., Honscheid, K., et al. 2015, AJ, 150, 150

Fong, W., Berger, E., Margutti, R., \& Zauderer, B. A. 2015, ApJ, 815, 102

Foucart, F. 2012, PhRvD, 86, 124007

Fruscione, A., McDowell, J. C., Allen, G. E., et al. 2006, Proc. SPIE, 6270, $62701 \mathrm{~V}$

Glowacki, M., Allison, J. R., Sadler, E. M., Moss, V. A., \& Jarrett, T. H. 2017, arXiv: 1709.08634

Goldstein, D. A., Andreoni, I., Nugent, P. E., et al. 2019, ApJL, 881, L7 Hajela, A., Margutti, R., Alexander, K. D., et al. 2019, arXiv:1909.06393 Hancock, P. J., Trott, C. M., \& Hurley-Walker, N. 2018, PASA, 35, e011 Herner, K., Soares-Santos, M., Annis, J., \& Palmese, A. 2019, GCN, 25495, 1 Hobbs, G., Heywood, I., Bell, M. E., et al. 2016, MNRAS, 456, 3948

Hotokezaka, K., Nissanke, S., Hallinan, G., et al. 2016, ApJ, 831, 190

Hotokezaka, K., \& Piran, T. 2015, MNRAS, 450, 1430

Jaodand, A., Campana, S., Brightman, M., et al. 2019, GCN, 25822, 1

Johnston, S., Taylor, R., Bailes, M., et al. 2008, ExA, 22, 151

Kasen, D., Metzger, B., Barnes, J., Quataert, E., \& Ramirez-Ruiz, E. 2017, Natur, 551, 80

Kauffmann, G., Heckman, T. M., Tremonti, C., et al. 2003, MNRAS, 346, 1055

Kocevski, D. 2019, GCN, 25326, 1

Kyutoku, K., Okawa, H., Shibata, M., \& Taniguchi, K. 2011, PhRvD, 84, 064018

Lamb, G. P., \& Kobayashi, S. 2016, ApJ, 829, 112 
Lang, D., Hogg, D. W., \& Mykytyn, D. 2016, The Tractor: Probabilistic Astronomical Source Detection and Measurement, Astrophysics Source Code Library, ascl:1604.008

LIGO Scientific Collaboration \& Virgo Collaboration et al. 2019a, GCN, 25324, 1

LIGO Scientific Collaboration \& Virgo Collaboration et al. 2019b, GCN, 25333, 1

McConnell, D., Allison, J. R., Bannister, K., et al. 2016, PASA, 33, e042

McMullin, J. P., Waters, B., Schiebel, D., Young, W., \& Golap, K. 2007, in ASP Conf. Ser. 376, Astronomical Data Analysis Software and Systems XVI, ed. R. A. Shaw, F. Hill, \& D. J. Bell (San Francisco, CA: ASP), 127 Molkov, S., Mereghetti, S., Savchenko, V., et al. 2019, GCN, 25323, 1

Mooley, K. P., Frail, D. A., Dobie, D., et al. 2018, ApJL, 868, L11

Mooley, K. P., Frail, D. A., Ofek, E. O., et al. 2013, ApJ, 768, 165

Oke, J. B., \& Gunn, J. E. 1982, PASP, 94, 586
Palmer, D. M., Barthelmy, S. D., Lien, A. Y., et al. 2019, GCN, 25341, 1

Pilia, M., Pittori, C., Tavani, M., et al. 2019, GCN, 25327, 1

Piran, T., Nakar, E., \& Rosswog, S. 2013, MNRAS, 430, 2121

Radcliffe, J. F., Beswick, R. J., Thomson, A. P., et al. 2019, MNRAS, 490, 4024

Rowlinson, A., Stewart, A. J., Broderick, J. W., et al. 2019, A\&C, 27, 111

Sugizaki, M., Kawai, N., Negoro, H., et al. 2019, GCN, 25329, 1

Swinbank, J. D., Staley, T. D., Molenaar, G. J., et al. 2015, A\&C, 11, 25

Whiting, M., Voronkov, M., Mitchell, D. \& Askap Team 2017, in ASP Conf. Ser. 512, Astronomical Data Analysis Software and Systems XXV, ed. N. P. F. Lorente, K. Shortridge, \& R. Wayth (San Francisco, CA: ASP), 431

Wilson, J. C., Eikenberry, S. S., Henderson, C. P., et al. 2003, Proc. SPIE, 4841, 451

Zhang, J., Yang, Y., Zhang, C., et al. 2019, MNRAS, 488, 5020 